\title{
ON ADDITIVE FUNCTIONS WITH ADDITIONAL DERIVATION PROPERTIES
}

\author{
RICHÁRD GRÜNWALD AND ZSOLT PÁLES
}

\begin{abstract}
The purpose of this paper is to introduce the notion of a generalized derivation which derivates a prescribed family of smooth vector-valued functions of several variables. The basic calculus rules are established and then a result derived which shows that if a function $f$ satisfies an addition theorem whose determining operation is derivable with respect to an additive function $d$, then the function $f$ is itself derivable with respect to $d$. As an application of this approach, new proof of a generalization of a recent result of Maksa is obtained. We also extend the result of Nishiyama and Horinouchi and formulate two open problems.
\end{abstract}

\section{INTRODUCTION}

Derivations are additive and Leibniz-type mappings of a ring into itself. More precisely, if $(R,+, \cdot)$ is a ring, then a function $d: R \rightarrow R$ is called a derivation if, for all $x, y \in R$,

$$
\begin{aligned}
d(x+y) & =d(x)+d(y), \\
d(x \cdot y) & =d(x) \cdot y+x \cdot d(y) .
\end{aligned}
$$

Derivations are used in many branches of analysis and algebra. For instance, nonnegative information functions are constructed via real derivations (see Daróczy-Maksa [4, Maksa [17]). Nonconstant functions that are convex with respect to families of power means are also obtained in terms of real derivations (see Maksa-Páles 21]). Derivations are used to express the general solutions of certain functional equations (see Fechner-Gselmann [5], Gselmann [7], 8], HalterKoch [12], [11], Jurkat [13]). Generalizations, such as higher-order derivations, bi-derivations and approximate or near-derivations were studied by Badora [1], Gselmann [9], Gselmann-Páles [10], and Maksa [18], [19].

We say that a function $d: \mathbb{R} \rightarrow \mathbb{R}$ derivates a differentiable function $f: I \rightarrow \mathbb{R}$ if the functional equation

$$
d(f(x))=f^{\prime}(x) d(x) \quad(x \in I)
$$

holds. In the pioneering papers [15, 16] Kurepa proved that if $d$ is an additive functions which derivates one of the maps $x \mapsto x^{2}$ or $x \mapsto x^{-1}$, then it satisfies the Leibniz Rule, i.e., it is a

Date: June 22, 2020.

2010 Mathematics Subject Classification. Primary 39B22, 39B40, 39B50.

Key words and phrases. algebraic derivation; derivation for trigonometric functions; derivation for hyperbolic functions.

The research of the first author was supported by the UNKP-19-2 New National Excellence Program of the Ministry of Human Capacities. The research of the second author was supported by the 2019-2.1.11-TÉT-201900049, the EFOP-3.6.1-16-2016-00022 and the EFOP-3.6.2-16-2017-00015 projects. The last two projects are co-financed by the European Union and the European Social Fund. 
standard derivation. This result was then extended by Nishiyama and Horinouchi in [22, who proved an analogous statement about the derivability of the power function $x \mapsto x^{r}$ with rational exponent different from 0 and 1. Boros and Erdei in [2] proved that those additive functions that derivate the map $x \mapsto \sqrt{1-x^{2}}$, that is, satisfy the identity

$$
d\left(\sqrt{1-x^{2}}\right)=-\frac{x}{\sqrt{1-x^{2}}} d(x) \quad(x \in]-1,1[),
$$

are also standard derivations. Maksa in [20] showed that if an additive function derivates any of the exponential, hyperbolic or trigonometric functions, then is has to be a standard derivation again. A counterpart of this result was obtained by Grünwald and Páles in [6], where an analogous statement was established assuming Leibniz property instead of additivity.

The purpose of this paper is to introduce the notion of generalized derivation which derivates a prescribed family of smooth vector-valued functions of several variables. After establishing the basic calculus rules in Theorem 1, we derive in Corollary 2 a result which shows that if a function $f$ satisfies an addition theorem whose determining operation is derivable with respect to an additive function $d$, then the function $f$ is itself derivable with respect to $d$. Using this result, we will be able to give a completely new proof for the aforementioned result of Maksa [20]. In addition, we also generalize this result, because we require the derivability of the exponential, hyperbolic or trigonometric functions only on small intervals. In the last section of our paper, we also offer a generalization of Nishiyama and Horinouchi by replacing power functions of the

form $P \circ Q^{-1}$, where $P$ and $Q$ are polynomials with rational coefficients. Finally, we formulate some open problems.

\section{Generalized Derivations And their properties}

For fixed $n, m \in \mathbb{N}$, the class of $n$-variable $\mathbb{R}^{m}$-valued admissible functions is defined as follows:

$$
\mathcal{A}_{n}^{m}:=\left\{f: \Omega \rightarrow \mathbb{R}^{m} \mid \emptyset \neq \Omega \subset \mathbb{R}^{n} \text { is open and } f \text { is Fréchet differentiable on } \Omega\right\}
$$

and we set

$$
\mathcal{A}:=\bigcup_{n, m=1}^{\infty} \mathcal{A}_{n}^{m} .
$$

The set $\Omega$ related to $f$ will be called the domain of $f$ and denoted by $\operatorname{dom}_{f}$. In general, for a vector $x \in \mathbb{R}^{n}$, we will denote the $i$ th coordinate of $x$ by $x_{i}$, and for a function $f \in \mathcal{A}_{n}^{m}$, $f_{j}$ will stand for the $j$ th coordinate function of $f$.

We say that a function $d: \mathbb{R} \rightarrow \mathbb{R}$ is a derivation with respect to an admissible function $f \in \mathcal{A}_{n}^{m}$ if, for all $x \in \operatorname{dom}_{f}$ and $j \in\{1, \ldots, m\}$,

$$
d\left(f_{j}(x)\right)=\partial_{1} f_{j}(x) d\left(x_{1}\right)+\cdots+\partial_{n} f_{j}(x) d\left(x_{n}\right)
$$

holds. Furthermore we say that $d$ is a derivation with respect to $A \subseteq \mathcal{A}$, if $d$ is a derivation with respect to each member of $A$. For any $n \in \mathbb{N}$ and $x \in \mathbb{R}^{n}$, define $d(x)$ by

$$
d(x):=\left(d\left(x_{1}\right), \ldots, d\left(x_{n}\right)\right) .
$$

Then (4) can simply be rewritten as

$$
d(f(x))=f^{\prime}(x) d(x)
$$


where $f \in \mathcal{A}_{n}^{m}$ and $f^{\prime}(x)$ denotes the Fréchet derivative of $f$ at $x$, which is an $n \times m$ matrix whose entries are the partial derivatives $\partial_{i} f_{j}$ at $x$.

One can immediately see that a function $d: \mathbb{R} \rightarrow \mathbb{R}$ is a standard derivation if and only if it is a derivation with respect to $S_{2}$ and $P_{2}$, where

$$
S_{2}\left(x_{1}, x_{2}\right):=x_{1}+x_{2} \quad \text { and } \quad P_{2}\left(x_{1}, x_{2}\right):=x_{1} x_{2} \quad\left(\left(x_{1}, x_{2}\right) \in \mathbb{R}^{2}\right) .
$$

In what follows, we will prefer the terminology $d$ is additive (resp. of Leibniz-type) whenever $d$ is a derivation with respect to $S_{2}$ (resp. $P_{2}$ ).

The following result, which is a significant extension of [6, Lemma A] collects the basic rules for functions that are derivable with respect to a fixed real function. In particular, its second assertion will be very useful for our purposes.

Theorem 1. For any function $d: \mathbb{R} \rightarrow \mathbb{R}$, we have the following three assertions.

(i) Let $n, m, k \in \mathbb{N}$ and $f \in \mathcal{A}_{n}^{m}, g \in \mathbb{R}_{m}^{k}$. If $d$ is a derivation with respect to $f$ and $g$, then $d$ is also a derivation with respect to $g \circ f$.

(ii) Let $n, m, k \in \mathbb{N}$ and $f \in \mathcal{A}_{n}^{m}, g \in \mathbb{R}_{m}^{k}$ such that $f\left(\operatorname{dom}_{f}\right)$ is open. If $d$ is a derivation with respect to $f$ and $g \circ f$, then $d$ is also a derivation with respect to $g$ on $f\left(\operatorname{dom}_{f}\right) \cap \operatorname{dom}_{g}$.

(iii) Let $n \in \mathbb{N}$ and $f \in \mathcal{A}_{n}^{n}$ with a continuous nowhere singular derivative. If $d$ is a derivation with respect to $f$, then $d$ is also a derivation with respect to its inverse $f^{-1}$.

Proof. By the assumptions of (i), for all $x \in \operatorname{dom}_{f}$ and $y \in \operatorname{dom}_{g}$, we have

$$
d(f(x))=f^{\prime}(x) d(x) \quad \text { and } \quad d(g(y))=g^{\prime}(y) d(y) .
$$

Let $x \in \operatorname{dom}_{f}$ with $y:=f(x) \in \operatorname{dom}_{g}$. Using that $d$ is a derivation with respect to $f$ and $g$, by the standard Chain Rule, we get

$$
\begin{aligned}
d((g \circ f)(x)) & =d(g(f(x)))=d(g(y))=g^{\prime}(y) d(y) \\
& =g^{\prime}(f(x)) d(f(x))=g^{\prime}(f(x)) f^{\prime}(x) d(x)=(g \circ f)^{\prime}(x) d(x),
\end{aligned}
$$

which yields that $d$ is a derivation with respect to the function $g \circ f$.

Let $y \in f\left(\operatorname{dom}_{f}\right) \cap \operatorname{dom}_{g}$. Then there exists $x \in \operatorname{dom}_{f}$ such that $y=f(x)$. Thus, applying the standard Chain Rule, we get

$$
\begin{aligned}
d(g(y)) & =d\left(g(f(x))=d(g \circ f(x))=(g \circ f)^{\prime}(x) d(x)\right. \\
& =g^{\prime}(f(x)) f^{\prime}(x) d(x)=g^{\prime}(f(x)) d(f(x))=g^{\prime}(y) d(y),
\end{aligned}
$$

which proves that $d$ is also a derivation with respect to $g$ on $f\left(\operatorname{dom}_{f}\right) \cap \operatorname{dom}_{g}$.

By the assumption of (iii), for all $x \in \operatorname{dom}_{f}$, we have the first equality in (5). Let $y \in \operatorname{dom}_{f^{-1}}$. Using the substitution $x=f^{-1}(y)$, this implies

$$
d(y)=f^{\prime}\left(f^{-1}(y)\right) d\left(f^{-1}(y)\right) .
$$

Thus, by the inverse function theorem, it follows that

$$
d\left(f^{-1}(y)\right)=\left(f^{\prime}\left(f^{-1}(y)\right)\right)^{-1} d(y)=\left(f^{-1}\right)^{\prime}(y) d(y) .
$$

Thus, $d$ is a derivation with respect to the inverse function $f^{-1}$.

The following consequence of the above theorem will be useful in several proofs. 
Corollary 2. Let $\Omega_{1}, \Omega_{2} \subseteq \mathbb{R}^{n}$ be nonempty open sets, let $f: \Omega_{1} \cup \Omega_{2} \cup\left(\Omega_{1}+\Omega_{2}\right) \rightarrow \mathbb{R}^{m}$ be a Fréchet differentiable function such that $f\left(\Omega_{1}\right)$ and $f\left(\Omega_{2}\right)$ are open. Assume that there exists a Fréchet differentiable function $g: f\left(\Omega_{1}\right) \times f\left(\Omega_{2}\right) \rightarrow \mathbb{R}^{m}$ such that $f$ satisfies the functional equation

$$
f(x+y)=g(f(x), f(y)) \quad\left((x, y) \in \Omega_{1} \times \Omega_{2}\right) .
$$

Let $d: \mathbb{R} \rightarrow \mathbb{R}$ be an additive function which is a derivation with respect to $f$. Then $d$ is also a derivation with respect to $g$ on $f\left(\Omega_{1}\right) \times f\left(\Omega_{2}\right)$.

Proof. Assume that $d$ is an additive derivation with respect to $f$. By the additivity of $d$, we have that $d$ is a derivation with respect to the mapping

$$
\Omega_{1} \times \Omega_{2} \ni(x, y) \mapsto f(x+y) .
$$

Thus, the equality (6) implies that $d$ is a derivation with respect to the composition

$$
\Omega_{1} \times \Omega_{2} \ni(x, y) \mapsto g(f(x), f(y)) .
$$

On the other hand, $d$ is trivially a derivation with respect to the mapping

$$
\Omega_{1} \times \Omega_{2} \ni(x, y) \mapsto(f(x), f(y)) .
$$

Applying the second assertion of the previous theorem, now it follows that $d$ is also a derivation with respect to $g$ on $f\left(\Omega_{1}\right) \times f\left(\Omega_{2}\right)$.

\section{LoCALizATion THEOREMS}

In the sequel, for a number $r \in \mathbb{Q}$, let $D_{r}$ denote the domain of the power function $x \mapsto x^{r}$, which is defined in the following way: If $r=m / n$, where $n \in \mathbb{N}, m \in \mathbb{Z}$ and $n, m$ are coprime, then let

$$
D_{r}:= \begin{cases}\mathbb{R} & \text { if } n \text { is odd and } m \geq 0, \\ \mathbb{R} \backslash\{0\} & \text { if } n \text { is odd and } m<0, \\ {[0, \infty[} & \text { if } n \text { is even and } m \geq 0, \\ ] 0, \infty[ & \text { if } n \text { is even and } m<0 .\end{cases}
$$

A function $d: \mathbb{R} \rightarrow \mathbb{R}$ is said to be $\mathbb{Q}$-homogeneous if, for all $x \in \mathbb{R}$ and $r \in \mathbb{Q}$, the equality $d(r x)=r d(x)$ holds. It is well-known that every additive function is automatically $\mathbb{Q}$ homogeneous.

Lemma 3. Let $r \in \mathbb{Q}$, let $I \subseteq D_{r-1}$ be a nonempty open subset and $d: \mathbb{R} \rightarrow \mathbb{R}$ be $a \mathbb{Q}$ homogeneous function. Suppose that the equality

$$
d\left(x^{r}\right)=r x^{r-1} d(x)
$$

holds for all $x \in I$. Then it is also valid for all $x \in D_{r-1}$.

Proof. Assume that (7) holds for all $x \in I$. Let $x \in D_{r-1}$ be arbitrary. If $x=0$, then $r \geq 1$ and hence $x^{r}=0^{r}=0$. Thus, by $d(0)=0$, (17) is trivially valid. If $r=1$ So may assume that $x$ is an arbitrarily fixed element from $D_{r} \backslash\{0\}$. Suppose that $r$ is of the form $r=m / n$ for some $n \in \mathbb{N}$ and $m \in \mathbb{Z}$. By the density of $\mathbb{Q}$ in $\mathbb{R}$, it is clear that the set

$$
\left\{q \in \mathbb{Q} \mid q \neq 0, q^{n} x \in I\right\}
$$


is nonempty. Let $q$ be a fixed element from it. Thus, using the validity of equation (7) on the interval $I$ and the $\mathbb{Q}$-homogeneity of $d$, we obtain

$$
q^{m} d\left(x^{r}\right)=d\left(q^{m} x^{r}\right)=d\left(\left(q^{n} x\right)^{r}\right)=r\left(q^{n} x\right)^{r-1} d\left(q^{n} x\right)=q^{m} r x^{r-1} d(x),
$$

which simplifies to $d\left(x^{r}\right)=r x^{r-1} d(x)$. This is exactly the desired equality since $x$ was an arbitrary element from $D_{r} \backslash\{0\}$.

The following result essentially was proved by Nishiyama and Horinouchi [22. The result concerning the particular cases $r=-1$ and $r=2$ were discovered by Kurepa in [15] and [16].

Lemma 4. Let $d: \mathbb{R} \rightarrow \mathbb{R}$ be an additive function, let $r \in \mathbb{Q} \backslash\{0,1\}$ and let $I \subseteq D_{r}$ be a nonempty open subinterval. Then $d$ is a standard derivation if and only if (7) is valid for all $x \in I$.

Proof. Assume first that $d$ is a standard derivation. Then by an easy argument, it follows that (17) is valid for all $x \in D_{r}$.

Conversely, if (7) is valid for all $x \in I$, then, by Lemma 3, we get that (7) is valid for all $x \in D_{r}$. Now, the result of Nishiyama and Horinouchi [22] implies that $d$ must be a standard derivation.

Lemma 5. Let $U \subseteq \mathbb{R}^{2}$ be a nonempty open subset and let $d: \mathbb{R} \rightarrow \mathbb{R}$ be a $\mathbb{Q}$-homogeneous function which satisfies the functional equation (2) for all $(x, y) \in U$. Then (2) also holds for all $x, y \in \mathbb{R}$.

Proof. Let $x, y \in \mathbb{R}$. Using the density of $\mathbb{Q}$ in $\mathbb{R}$, it is clear that there exist $p, q \in \mathbb{Q} \backslash\{0\}$ such that $(p x, q y) \in U$. Applying equation (2) for $p x$ and $q y$, taking into consideration the $\mathbb{Q}$-homogeneity of $d$, we obtain that

$$
p q d(x y)=d((p x)(q y))=d(p x) q y+p x d(q y)=p d(x) q y+p x q d(y) .
$$

Dividing by $p q$ the above equality, we get the statement.

To prove our main result, which will extend the theorem of Maksa [20], the following lemma will also be needed.

Lemma 6. The sets

$$
\begin{aligned}
U & :=\left\{x \in \mathbb{R}: x, \sqrt{1+x^{2}} \in \mathbb{Q}\right\} \\
V & :=\{x \in]-\infty,-1[\cup] 1, \infty\left[: x, \sqrt{x^{2}-1} \in \mathbb{Q}\right\}, \\
W & :=\{x \in]-1,1\left[: x, \sqrt{1-x^{2}} \in \mathbb{Q}\right\}
\end{aligned}
$$

are dense in $\mathbb{R}$, in $]-\infty,-1[\cup] 1, \infty[$, and in $]-1,1[$, respectively.

Proof. To prove the density of $U$, let $x \in]-1,1[$, let $0<\varepsilon<\min (1+x, 1-x)$ be arbitrary and denote

We are going to show that

$$
I:=] \sqrt{\frac{1-x-\varepsilon}{1+x+\varepsilon}}, \sqrt{\frac{1-x+\varepsilon}{1+x-\varepsilon}}[.
$$

$$
\left.\frac{1-r^{2}}{1+r^{2}} \in\right] x-\varepsilon, x+\varepsilon[\cap U \quad \text { for all } \quad r \in \mathbb{Q} \cap I .
$$


Indeed, let $r \in \mathbb{Q} \cap I$ be arbitrary. Then there exists $(m, n) \in \mathbb{Z} \times \mathbb{N}$ such that $r=\frac{m}{n}$. Using that $r$ is bounded by the endpoints of $I$, we easily get that

$$
\left.s:=\frac{1-r^{2}}{1+r^{2}} \in\right] x-\varepsilon, x+\varepsilon[\text {. }
$$

On the other hand, $s \in \mathbb{Q}$ and

$$
\sqrt{1-s^{2}}=\sqrt{1-\left(\frac{1-r^{2}}{1+r^{2}}\right)^{2}}=\sqrt{1-\left(\frac{n^{2}-m^{2}}{n^{2}+m^{2}}\right)^{2}}=\frac{2 n m}{n^{2}+m^{2}} \in \mathbb{Q},
$$

which completes the proof of (8) .

To prove the density of $V$, let $x \in]-\infty,-1[\cup] 1, \infty[$, that is, let $|x|>1$, let $0<\varepsilon<|x|-1$ be arbitrary and denote

$$
J:= \begin{cases}] \sqrt{\frac{x+\varepsilon-1}{x+\varepsilon+1}}, \sqrt{\frac{x-\varepsilon-1}{x-\varepsilon+1}}[ & \text { if } x<-1, \\ ] \sqrt{\frac{x-\varepsilon-1}{x-\varepsilon+1}}, \sqrt{\frac{x+\varepsilon-1}{x+\varepsilon+1}}[ & \text { if } 1<x .\end{cases}
$$

Then, one can easily check that $J$ is nonempty and $J \subseteq] 1, \infty[$ if $x<-1$ and $J \subseteq] 0,1[$ if $1<x$. Thus $1 \notin J$ holds in both cases. We are going to show that

$$
\left.\frac{1+r^{2}}{1-r^{2}} \in\right] x-\varepsilon, x+\varepsilon[\cap V \quad \text { for all } \quad r \in \mathbb{Q} \cap J .
$$

Indeed, let $r \in \mathbb{Q} \cap J$ be arbitrary. Then there exists $(m, n) \in \mathbb{N} \times \mathbb{N}$ such that $r=\frac{m}{n}$. Since $r$ cannot be equal to 1 , therefore $n \neq m$ holds. Using that $r$ is bounded by the endpoints of $J$, in each cases we easily get that

$$
\left.s:=\frac{1+r^{2}}{1-r^{2}} \in\right] x-\varepsilon, x+\varepsilon[
$$

On the other hand, $s \in \mathbb{Q}$ and

$$
\sqrt{s^{2}-1}=\sqrt{\left(\frac{1+r^{2}}{1-r^{2}}\right)^{2}-1}=\sqrt{\left(\frac{n^{2}+m^{2}}{n^{2}-m^{2}}\right)^{2}-1}=\frac{2 n m}{\left|n^{2}-m^{2}\right|} \in \mathbb{Q}
$$

which completes the proof of (9)).

To prove the density of $W$, let $x \in \mathbb{R} \backslash\{0\}$ and $0<\varepsilon<|x|$ be arbitrary and denote

$$
K:= \begin{cases}\int \frac{-1}{x+\varepsilon}-\sqrt{\frac{1}{(x+\varepsilon)^{2}}+1}, 1[ & \text { if } x<0, \\ ]-1, \frac{-1}{x+\varepsilon}+\sqrt{\frac{1}{(x+\varepsilon)^{2}}+1}[\quad \text { if } 0<x .\end{cases}
$$

Then, one can easily check that $K$ is nonempty and $K \subseteq]-1,1[$. Thus $1 \notin K$ holds in both cases. We are going to show that

$$
\left.\frac{2 r}{1-r^{2}} \in\right] x-\varepsilon, x+\varepsilon[\cap W \quad \text { for all } \quad r \in \mathbb{Q} \cap K .
$$


Indeed, let $r \in \mathbb{Q} \cap K$ be arbitrary. Then there exists $(m, n) \in \mathbb{Z} \times \mathbb{N}$ such that $r=\frac{m}{n}$. Since $r$ cannot be equal to 1 , therefore $n \neq m$ holds. Using that $r$ is bounded by the endpoints of $K$, in each cases we easily get that

$$
\left.s:=\frac{2 r}{1-r^{2}} \in\right] x-\varepsilon, x+\varepsilon[.
$$

On the other hand, $s \in \mathbb{Q}$ and

$$
\sqrt{1+s^{2}}=\sqrt{1+\left(\frac{2 r}{1-r^{2}}\right)^{2}}=\sqrt{1+\left(\frac{2 n m}{n^{2}-m^{2}}\right)^{2}}=\frac{n^{2}+m^{2}}{\left|n^{2}-m^{2}\right|} \in \mathbb{Q},
$$

which completes the proof of (10).

\section{Extension of the Result of Maksa}

In what follows we extend the result of Maksa [20] by assuming the derivability of any of the exponential, hyperbolic or trigonometric functions on a small interval. Our approach is based on the use of the addition theorems for each of these functions and the application Corollary 2 , In each particular case, we obtain that $d$ is a derivation with respect to a two-variable algebraic function.

Theorem 7. Let $d: \mathbb{R} \rightarrow \mathbb{R}$ be an additive function and let $\alpha, \beta \in \mathbb{R}$ with $\alpha<\beta$. Suppose that $d$ is a derivation with respect to the restriction to $] \alpha, \beta[$ of any of the following functions with further assumptions on $\alpha$ and $\beta$, respectively:

$\begin{array}{rll}\text { (i) } & \text { exp } & (2 \alpha<\beta \text { and } \alpha<2 \beta), \\ (\text { ii }) & \text { sinh } & (\alpha<0<\beta), \\ (\text { iii }) & \text { cosh } & (0<2 \alpha<\beta \text { or } \alpha<2 \beta<0), \\ (\text { iv }) & \text { tanh } & (\alpha<0<\beta), \\ (\text { v }) & \text { coth } & (0<2 \alpha<\beta \text { or } \alpha<2 \beta<0), \\ (\text { vi }) & \text { sin } & (\alpha<0<\beta), \\ (\text { vii }) & \cos & (0<2 \alpha<\pi<\beta \text { or } \alpha<-\pi<2 \beta<0), \\ \text { (viii }) & \text { tan } & (-\pi<2 \alpha<\beta \text { and } \alpha<2 \beta<\pi), \\ (\text { ix }) & \cot & (0<2 \alpha<\beta<\pi \text { or }-\pi<\alpha<2 \beta<0) .\end{array}$

Then $d$ is a standard derivation.

Proof. Observe that in each of the above cases the inequalities $2 \alpha<\beta$ and $\alpha<2 \beta$ hold. Adding up these inequalities side by side, it follows that $\alpha<\beta$ and hence

$$
\gamma:=\frac{1}{2} \max (\alpha, 2 \alpha)<\frac{1}{2} \min (\beta, 2 \beta)=: \delta .
$$

Then $] \gamma, \delta[\subseteq] \alpha, \beta[\cap] \frac{\alpha}{2}, \frac{\beta}{2}[$, which implies that

$$
] \gamma, \delta[+] \gamma, \delta[=] 2 \gamma, 2 \delta[\subseteq] \alpha, \beta[.
$$

In the rest of proof, we shall utilize that each of the functions listed in (11) possesses an addition formula, i.e., it satisfies functional equation of type (6) with $\left.\Omega_{1}:=\Omega_{2}:=\right] \gamma, \delta[$. 
(i) Assume first that $2 \alpha<\beta$ and $\alpha<2 \beta$ and $d$ is a derivation with respect to the restriction to $] \alpha, \beta[$ of the exponential function. It means that the equation $d(\exp (x))=\exp (x) d(x)$ holds for all $x \in] \alpha, \beta[$. Using that this restriction satisfies the functional equation

$$
\exp (x+y)=\exp (x) \exp (y) \quad(x, y \in] \gamma, \delta[)
$$

Corollary 2 implies that $d$ is a derivation with respect to the mapping

$$
(u, v) \mapsto u \cdot v \quad(u, v \in] \exp (\gamma), \exp (\delta)[),
$$

i.e., $d$ is of Leibniz-type on the interval $] \exp (\gamma), \exp (\delta)$ [. In view of Lemma 5, it follows that $d$ is of Leibniz-type on $\mathbb{R}$ and hence it is a standard derivation.

(ii) In the second case assume that $\alpha<0<\beta$ and $d(\sinh (x))=\cosh (x) d(x)$ holds for all $x \in] \alpha, \beta\left[\right.$. Then $\gamma=\frac{\alpha}{2}$ and $\delta=\frac{\beta}{2}$. First we can choose $\lambda>0$ such that $]-\lambda, \lambda[\subseteq] \gamma, \delta[$. Using the identity $\cosh (x)=\sqrt{1+\sinh ^{2}(x)}(x \in]-\lambda, \lambda[)$, we obtain that the restriction of the sine hyperbolic function to the interval $] \alpha, \beta[$ satisfies the functional equation

$$
\sinh (x+y)=\sinh (x) \sqrt{1+\sinh ^{2}(y)}+\sqrt{1+\sinh ^{2}(x)} \sinh (y)
$$

for all $x, y \in]-\lambda, \lambda[$. By Corollary 2 , it follows that $d$ is also a derivation with respect to the function

$$
(u, v) \mapsto u \sqrt{1+v^{2}}+v \sqrt{1+u^{2}} \quad(u, v \in]-\sinh (\lambda), \sinh (\lambda)[) .
$$

It means that the functional equation

$$
\begin{aligned}
& d\left(u \sqrt{1+v^{2}}+v \sqrt{1+u^{2}}\right) \\
& \quad=\left(\sqrt{1+v^{2}}+v \frac{u}{\sqrt{1+u^{2}}}\right) d(u)+\left(\sqrt{1+u^{2}}+u \frac{v}{\sqrt{1+v^{2}}}\right) d(v)
\end{aligned}
$$

holds for all $u, v \in]-\sinh (\lambda), \sinh (\lambda)[$. Replacing $v$ by $-v$ and adding the equality so obtained to (12) side by side, we get that

$$
d\left(u \sqrt{1+v^{2}}\right)=\sqrt{1+v^{2}} d(u)+u \frac{v}{\sqrt{1+v^{2}}} d(v)
$$

holds for all $u, v \in]-\sinh (\lambda), \sinh (\lambda)[$. Let $U$ be the set defined in Lemma 6. Then, by this lemma, the intersection $U \cap]-\sinh (\lambda), \sinh (\lambda)[$ is nonempty, moreover it is dense in ] $\sinh (\lambda), \sinh (\lambda)[$. Then, for $u, v \in U \cap]-\sinh (\lambda), \sinh (\lambda)\left[\right.$, we have that $u, v$, and $\sqrt{1+v^{2}} \in \mathbb{Q}$. For such values of $u$ and $v$, the equality (13) and the $\mathbb{Q}$-homogeneity of $d$ implies that

$$
u \sqrt{1+v^{2}} d(1)=\sqrt{1+v^{2}} u d(1)+u \frac{v}{\sqrt{1+v^{2}}} v d(1),
$$

which is possible only if $d(1)=0$. Let $0 \neq u \in]-\sinh (\lambda), \sinh (\lambda)[\cap \mathbb{Q}$, and $v \in]-\sinh (\lambda), \sinh (\lambda)[$. By the $\mathbb{Q}$-homogeneity and additivity of $d$, it follows that $d(u)=u d(1)=0$ and hence (13) simplifies to (3) on the interval ] $-\sinh (\lambda), \sinh (\lambda)[$. Using this, (13) can be rewritten as

$$
d\left(u \sqrt{1+v^{2}}\right)=\sqrt{1+v^{2}} d(u)+u d\left(\sqrt{1+v^{2}}\right)
$$

for all $u, v \in]-\sinh (\lambda), \sinh (\lambda)\left[\right.$. With the substitution $w:=\sqrt{1+v^{2}}$, this equality yields that

$$
d(u w)=u d(w)+w d(u)
$$


for all $u \in]-\sinh (\lambda), \sinh (\lambda)[$ and $w \in[1, \cosh (\lambda)[$. Then, in view of Lemma 5 , $d$ is of Leibniztype and hence is a standard derivation on $\mathbb{R}$.

(iii) In the third case suppose that $0<2 \alpha<\beta$ (the other case can be treated similarly) and $d(\cosh (x))=\sinh (x) d(x)$ holds for all $x \in] \alpha, \beta\left[\right.$. Then $\gamma=\alpha$ and $\delta=\frac{\beta}{2}$. The restriction of the cosine hyperbolic function to the interval $] \alpha, \beta[$ satisfies the functional equation

$$
\cosh (x+y)=\cosh (x) \cosh (y)+\sqrt{\cosh ^{2}(x)-1} \sqrt{\cosh ^{2}(y)-1}
$$

for all $x, y \in] \gamma, \delta[$. By Corollary 2 , it follows that $d$ is also a derivation with respect to the function

$$
(u, v) \mapsto u v+\sqrt{u^{2}-1} \sqrt{v^{2}-1} \quad(u, v \in] \cosh (\gamma), \cosh (\delta)[),
$$

i.e., the functional equation

$$
d\left(u v+\sqrt{u^{2}-1} \sqrt{v^{2}-1}\right)=\left(v+u \frac{\sqrt{v^{2}-1}}{\sqrt{u^{2}-1}}\right) d(u)+\left(u+v \frac{\sqrt{u^{2}-1}}{\sqrt{v^{2}-1}}\right) d(v)
$$

holds for all $u, v \in] \cosh (\gamma), \cosh (\delta)[$. Let $V$ be the set defined in Lemma 6. Then, by this lemma, the intersection $V \cap] \cosh (\gamma), \cosh (\delta)$ [ is nonempty, moreover it is dense in $] \cosh (\gamma), \cosh (\delta)[$. Then, for $u, v \in V \cap] \cosh (\gamma), \cosh (\delta)\left[\right.$, we have that $u, v$, and $\sqrt{v^{2}-1} \in \mathbb{Q}$. For such values of $u$ and $v$, the equality (14) and the $\mathbb{Q}$-homogeneity of $d$ implies that

$$
\begin{aligned}
\left(u v+\sqrt{u^{2}-1}\right. & \left.\sqrt{v^{2}-1}\right) d(1) \\
& =\left(v+u \frac{\sqrt{v^{2}-1}}{\sqrt{u^{2}-1}}\right) u d(1)+\left(u+v \frac{\sqrt{u^{2}-1}}{\sqrt{v^{2}-1}}\right) v d(1),
\end{aligned}
$$

which is possible only if $d(1)=0$. Substituting $v:=u$ in (14), using the additivity and $\mathbb{Q}$ homogeneity of $d$ and that $d(1)=0$ we get that (7) with $r=2$ holds for all $u \in] \cosh (\gamma), \cosh (\delta)[$, which, using Lemma 4, implies that $d$ is a standard derivation.

(iv) In the fourth case assume that $\alpha<0<\beta$ and $d(\tanh (x))=\frac{1}{\cosh ^{2}(x)} d(x)$ is valid for all $x \in] \alpha, \beta\left[\right.$. Then $\gamma=\frac{\alpha}{2}$ and $\delta=\frac{\beta}{2}$. The restriction of the tangent hyperbolic function to the interval $] \alpha, \beta[$ satisfies the functional equation

$$
\tanh (x+y)=\frac{\tanh (x)+\tanh (y)}{1+\tanh (x) \tanh (y)} \quad(x, y \in] \gamma, \delta[) .
$$

By Corollary 2, it follows that $d$ is also a derivation with respect to the function

$$
(u, v) \mapsto \frac{u+v}{1+u v} \quad(u, v \in] \tanh (\gamma), \tanh (\delta)[),
$$

i.e., the functional equation

$$
d\left(\frac{u+v}{1+u v}\right)=\frac{1-v^{2}}{(1+u v)^{2}} d(u)+\frac{1-u^{2}}{(1+u v)^{2}} d(v)
$$

holds for all $u, v \in] \tanh (\gamma), \tanh (\delta)[$. Now choose a subinterval $] \lambda, \mu[$ of $] \tanh (\gamma), \tanh (\delta)[$ such that $\lambda, \mu \in \mathbb{Q}$ and $0<\lambda \mu=: r$. It is easy to see that if $u \in] \lambda, \mu\left[\right.$, then $\left.\frac{r}{u} \in\right] \lambda, \mu[$ also 
holds. Substituting $u \in] \lambda, \mu\left[\right.$ and $v:=\frac{r}{u}$, (15) implies

$$
\frac{1}{1+r} d\left(u+\frac{r}{u}\right)=\frac{1-\left(\frac{r}{u}\right)^{2}}{(1+r)^{2}} d(u)+\frac{1-u^{2}}{(1+r)^{2}} d\left(\frac{r}{u}\right) .
$$

A direct and simple computation yields that

$$
-\frac{1}{u^{2}} d(u)=d\left(\frac{1}{u}\right)
$$

for $u \in] \lambda, \mu[$, which, using Lemma 4, implies that $d$ is a standard derivation.

$(v)$ In the fifth case assume that $0<2 \alpha<\beta$ (the other case can be handled similarly) and $d(\operatorname{coth}(x))=-\frac{1}{\sinh ^{2}(x)} d(x)$ holds for all $\left.x \in\right] \alpha, \beta\left[\right.$. Then $\gamma=\frac{\alpha}{2}$ and $\delta=\frac{\beta}{2}$. The restriction of the cotangent hyperbolic function to the interval ] $\alpha, \beta[$ satisfies the functional equation

$$
\operatorname{coth}(x+y)=\frac{\operatorname{coth}(x) \operatorname{coth}(y)+1}{\operatorname{coth}(x)+\operatorname{coth}(y)} \quad(x, y \in] \gamma, \delta[) .
$$

By Corollary 2, it follows that $d$ is also a derivation with respect to the function

$$
(u, v) \mapsto \frac{u v+1}{u+v} \quad(u, v \in] \operatorname{coth}(\delta), \operatorname{coth}(\gamma)[)
$$

i.e., the functional equation

$$
d\left(\frac{u v+1}{u+v}\right)=\frac{v^{2}-1}{(u+v)^{2}} d(u)+\frac{u^{2}-1}{(u+v)^{2}} d(v)
$$

holds for all $u, v \in] \operatorname{coth}(\delta), \operatorname{coth}(\gamma)[$. Now choose a subinterval $] \lambda, \mu[$ of $] \operatorname{coth}(\delta), \operatorname{coth}(\gamma)[\operatorname{such}$ that $\lambda, \mu \in \mathbb{Q}$ and $\lambda+\mu=: r \neq 0$. It is easy to see that if $u \in] \lambda, \mu[$, then $r-u \in] \lambda, \mu[$ also holds. Substituting $u \in] \lambda, \mu[$ and $v:=r-u$, (16) implies

$$
\frac{1}{r} d(u(r-u)+1)=\frac{(r-u)^{2}-1}{r^{2}} d(u)+\frac{u^{2}-1}{r^{2}} d(r-u) .
$$

This equality, using the additivity and $\mathbb{Q}$-homogeneity, after some simplification, reduces to

$$
d\left(u^{2}\right)=2 u d(u)+2 d(1)-u^{2} d(1) \quad(u \in] \lambda, \mu[) .
$$

If $u$ is rational, then this equality gives that $d(1)=0$. Hence the above equality shows that (7) is valid on $] \lambda, \mu$ [ with $r=2$. In view of Lemma 4, we obtain that $d$ is a standard derivation.

(vi) In the sixth case assume that $\alpha<0<\beta$ and $d(\sin (x))=\cos (x) d(x)$ holds for all $x \in] \alpha, \beta[$. Then $\gamma=\frac{\alpha}{2}$ and $\delta=\frac{\beta}{2}$. First we can choose $\lambda>0$ such that $]-\lambda, \lambda[\subseteq] \gamma, \delta[\cap]-\frac{\pi}{2}, \frac{\pi}{2}[$. Thus the cosine function is everywhere positive over $]-\lambda, \lambda[$. Then the sine function is strictly increasing on $]-\lambda, \lambda\left[\right.$ and $\cos (x)=\sqrt{1-\sin ^{2}(x)}$ holds for all $\left.x \in\right]-\lambda, \lambda[$. Therefore the restriction of the sine function to the interval $] \alpha, \beta$ [ satisfies the functional equation

$$
\sin (x+y)=\sin (x) \sqrt{1-\sin ^{2}(y)}+\sqrt{1-\sin ^{2}(x)} \sin (y) \quad(x, y \in]-\lambda, \lambda[) .
$$

By Corollary 2, it follows that $d$ is also a derivation with respect to the function

$$
(u, v) \mapsto u \sqrt{1-v^{2}}+v \sqrt{1-u^{2}} \quad(u, v \in]-\sin (\lambda), \sin (\lambda)[) .
$$


It means that the functional equation

$$
\begin{aligned}
& d\left(u \sqrt{1-v^{2}}+v \sqrt{1-u^{2}}\right) \\
& \quad=\left(\sqrt{1-v^{2}}-v \frac{u}{\sqrt{1-u^{2}}}\right) d(u)+\left(\sqrt{1-u^{2}}-u \frac{v}{\sqrt{1-v^{2}}}\right) d(v)
\end{aligned}
$$

holds for all $u, v \in]-\sin (\lambda), \sin (\lambda)[$. Replacing $v$ by $-v$ and adding the equality so obtained to (17) side by side, we get that

$$
d\left(u \sqrt{1-v^{2}}\right)=\sqrt{1-v^{2}} d(u)-u \frac{v}{\sqrt{1-v^{2}}} d(v)
$$

holds for all $u, v \in]-\sin (\lambda), \sin (\lambda)[$. Let $W$ be the set defined in Lemma 6 . Then, by this lemma, the intersection $W \cap]-\sin (\lambda), \sin (\lambda)[$ is nonempty, moreover it is dense in $]-\sin (\lambda), \sin (\lambda)[$. Then, for $u, v \in W \cap]-\sin (\lambda), \sin (\lambda)\left[\right.$, we have that $u, v$, and $\sqrt{1-v^{2}} \in \mathbb{Q}$. For such values of $u$ and $v$, the equality (18) and the $\mathbb{Q}$-homogeneity of $d$ implies that

$$
u \sqrt{1-v^{2}} d(1)=\sqrt{1-v^{2}} u d(1)-u \frac{v}{\sqrt{1-v^{2}}} v d(1),
$$

which is possible only if $d(1)=0$. Let $0 \neq u \in]-\sin (\lambda), \sin (\lambda)[\cap \mathbb{Q}$, and $v \in]-\sin (\lambda), \sin (\lambda)[$. By the $\mathbb{Q}$-homogeneity and additivity of $d$, it follows that $d(u)=u d(1)=0$ and hence (18) simplifies to (3) on the interval ] $-\sin (\lambda), \sin (\lambda)[$. Using this, (18) can be rewritten as

$$
d\left(u \sqrt{1-v^{2}}\right)=\sqrt{1-v^{2}} d(u)+u d\left(\sqrt{1-v^{2}}\right)
$$

for all $u, v \in]-\sin (\lambda), \sin (\lambda)\left[\right.$. With the substitution $w:=\sqrt{1-v^{2}}$, this equality yields that

$$
d(u w)=u d(w)+w d(u)
$$

for all $u \in]-\sin (\lambda), \sin (\lambda)[$ and $w \in] \cos (\lambda), 1]$. Then, in view of Lemma 5 , $d$ is of Leibniz-type and hence is a standard derivation on $\mathbb{R}$.

(vii) In the seventh case suppose that $0<2 \alpha<\pi<\beta$ (the other case can be treated similarly) and $d(\cos (x))=-\sin (x) d(x)$ holds for all $x \in] \alpha, \beta\left[\right.$. Then $\gamma=\alpha$ and $\delta=\frac{\beta}{2}$ and we can choose $\lambda \in] 0, \frac{\pi}{2}[$ such that $] \frac{\pi}{2}-\lambda, \frac{\pi}{2}+\lambda[\subseteq] \gamma, \delta[$. Thus the sine function is positive over $] \frac{\pi}{2}-\lambda, \frac{\pi}{2}+\lambda[$ and hence $\sin (x)=\sqrt{1-\cos ^{2}(x)}$ holds for all $\left.x \in\right] \frac{\pi}{2}-\lambda, \frac{\pi}{2}+\lambda[$. The restriction of the cosine function to the interval $] \alpha, \beta[$ satisfies the functional equation

$$
\cos (x+y)=\cos (x) \cos (y)-\sqrt{1-\cos ^{2}(x)} \sqrt{1-\cos ^{2}(y)}
$$

for all $x, y \in] \frac{\pi}{2}-\lambda, \frac{\pi}{2}+\lambda[$. By Corollary 2, it follows that $d$ is also a derivation with respect to the function

$$
(u, v) \mapsto u v-\sqrt{1-u^{2}} \sqrt{1-v^{2}} \quad(u, v \in] \cos \left(\frac{\pi}{2}+\lambda\right), \cos \left(\frac{\pi}{2}-\lambda\right)[),
$$

i.e., the functional equation

$$
d\left(u v-\sqrt{1-u^{2}} \sqrt{1-v^{2}}\right)=\left(v+u \frac{\sqrt{1-v^{2}}}{\sqrt{1-u^{2}}}\right) d(u)+\left(u+v \frac{\sqrt{1-u^{2}}}{\sqrt{1-v^{2}}}\right) d(v)
$$

holds for all $u, v \in] \cos \left(\frac{\pi}{2}+\lambda\right), \cos \left(\frac{\pi}{2}-\lambda\right)[$. Replacing $v$ by $-v$ and subtracting the equality so obtained from (19), we get that

$$
d(u v)=v d(u)+u d(v)
$$


holds for all $u, v \in] \cos \left(\frac{\pi}{2}+\lambda\right), \cos \left(\frac{\pi}{2}-\lambda\right)[$. Hence, in view of Lemma 5, we get that $d$ is a standard derivation on $\mathbb{R}$.

(viii) In the eighth case suppose that $-\pi<2 \alpha<\beta$ and $\alpha<2 \beta<\pi$ and $d(\tan (x))=$ $\frac{1}{\cos ^{2}(x)} d(x)$ is valid for all $\left.x \in\right] \alpha, \beta[$. The restriction of the tangent function to the interval $] \alpha, \beta[$ satisfies the functional equation

$$
\tan (x+y)=\frac{\tan (x)+\tan (y)}{1-\tan (x) \tan (y)} \quad(x, y \in] \gamma, \delta[) .
$$

By Corollary 2, it follows that $d$ is also a derivation with respect to the function

$$
(u, v) \mapsto \frac{u+v}{1-u v} \quad(u, v \in] \tan (\gamma), \tan (\delta)[),
$$

i.e., the functional equation

$$
d\left(\frac{u+v}{1-u v}\right)=\frac{1+v^{2}}{(1-u v)^{2}} d(u)+\frac{1+u^{2}}{(1-u v)^{2}} d(v)
$$

holds for all $u, v \in] \tan (\gamma), \tan (\delta)[$. Now choose a subinterval $] \lambda, \mu[$ of $] \tan (\gamma), \tan (\delta)[\operatorname{such}$ that $\lambda, \mu \in \mathbb{Q}$ and $0<\lambda \mu=: r \neq 1$. It is easy to see that if $u \in] \lambda, \mu\left[\right.$, then $\left.\frac{r}{u} \in\right] \lambda, \mu[$ also holds. Substituting $u \in] \lambda, \mu\left[\right.$ and $v:=\frac{r}{u}$, (21) implies

$$
\frac{1}{1-r} d\left(u+\frac{r}{u}\right)=\frac{1+\left(\frac{r}{u}\right)^{2}}{(1-r)^{2}} d(u)+\frac{1+u^{2}}{(1-r)^{2}} d\left(\frac{r}{u}\right) .
$$

A direct and simple computation yields that

$$
-\frac{1}{u^{2}} d(u)=d\left(\frac{1}{u}\right)
$$

for $u \in] \lambda, \mu[$, which, using Lemma 4, yields that $d$ is a standard derivation.

( $i x$ ) In the last case suppose that $0<2 \alpha<\beta<\pi$ (the case $-\pi<\alpha<2 \beta<0$ can be treated similarly) and $d(\cot (x))=-\frac{1}{\sin ^{2}(x)} d(x)$ is valid for all $\left.x \in\right] \alpha, \beta[$. The restriction of the cotangent function to the interval $] \alpha, \beta[$ satisfies the functional equation

$$
\cot (x+y)=\frac{\cot (x) \cot (y)-1}{\cot (x)+\cot (y)} \quad(x, y \in] \gamma, \delta[) .
$$

By Corollary 2, it follows that $d$ is also a derivation with respect to the function

$$
(u, v) \mapsto \frac{u v-1}{u+v} \quad(u, v \in] \cot (\delta), \cot (\gamma)[),
$$

i.e., the functional equation

$$
d\left(\frac{u v-1}{u+v}\right)=\frac{1+v^{2}}{(u+v)^{2}} d(u)+\frac{1+u^{2}}{(u+v)^{2}} d(v)
$$

holds for all $u, v \in] \cot (\delta), \cot (\gamma)[$. Now choose a subinterval $] \lambda, \mu[$ of $] \cot (\delta), \cot (\gamma)[\operatorname{such}$ that $\lambda, \mu \in \mathbb{Q}$ and $\lambda+\mu=: r \neq 0$. It is easy to see that if $u \in] \lambda, \mu[$, then $r-u \in] \lambda, \mu[$ also holds. Substituting $u \in] \lambda, \mu[$ and $v:=r-u$, (22) implies

$$
\frac{1}{r} d(u(r-u)-1)=\frac{1+(r-u)^{2}}{r^{2}} d(u)+\frac{1+u^{2}}{r^{2}} d(r-u) .
$$


This equality, using the additivity and $\mathbb{Q}$-homogeneity, after some simplification, reduces to

$$
d\left(u^{2}\right)=2 u d(u)-2 d(1)-u^{2} d(1) \quad(u \in] \lambda, \mu[) .
$$

If $u$ is rational, then this equality gives that $d(1)=0$. Hence the above equality shows that (7) is valid on $] \lambda, \mu[$ with $r=2$. In view of Lemma 4 we get that $d$ is a standard derivation.

When considering the interval $] \alpha, \beta[$, one should observe that depending on additional assumptions described in the nine cases of the theorem, this interval can be arbitrary small in all the cases except the case (vii), then it contains either $[\pi / 2, \pi]$ or $[-\pi,-\pi / 2]$.

\section{Extension of the Result of Nishiyama And Horinouchi}

Theorem 8. Let $d: \mathbb{R} \rightarrow \mathbb{R}$ be an additive function, $I$ be a nonempty open interval not containing zero and assume that $P, Q: I \rightarrow \mathbb{R}$ are of the form

$$
P(u)=\sum_{k \in \mathbb{Z}} p_{k} u^{k}, \quad Q(u)=\sum_{k \in \mathbb{Z}} q_{k} u^{k},
$$

where $p_{k}, q_{k} \in \mathbb{Q}$ for all $k \in \mathbb{Z}$ and the set $\left\{k \in \mathbb{Z} \mid\left(p_{k}, q_{k}\right) \neq(0,0)\right\}$ is finite. Then

$$
Q^{\prime}(u) d(P(u))=P^{\prime}(u) d(Q(u)) \quad(u \in I)
$$

holds if and only if

(i) either $P$ and $Q$ are linearly dependent,

(ii) or $P$ and $Q$ are linearly independent, $P-p_{0}$ and $Q-q_{0}$ are linearly dependent and $d(1)=0$, (iii) or $P-p_{0}$ and $Q-q_{0}$ are linearly independent and $d$ is a standard derivation.

Proof. First we prove the necessity of (i)-(iii). Assume that (24) holds and $P, Q$ are linearly independent. Then the Wronskian $P$ and $Q$ is not identically zero on $I$, i.e., there exists $u_{0} \in I$ such that $P^{\prime}\left(u_{0}\right) Q\left(u_{0}\right) \neq P\left(u_{0}\right) Q^{\prime}\left(u_{0}\right)$.

Substituting $u \in I \cap \mathbb{Q}$ we have that $P(u)$ and $Q(u)$ are rational numbers. Therefore, using the $\mathbb{Q}$-homogeneity of $d$, (24) implies that

$$
Q^{\prime}(u) P(u) d(1)=P^{\prime}(u) Q(u) u d(1)
$$

for all $u \in I \cap \mathbb{Q}$. By the continuity of $P$ and $Q$ and the density of $I \cap \mathbb{Q}$ in $I$, it follows that (25) holds for all $u \in I$, in particular, for $u=u_{0}$, which implies that $d(1)=0$.

From now on, we assume that $P-p_{0}$ and $Q-q_{0}$ are linearly independent. Let $k_{0}$ denote the smallest element of the finite set $\left\{k \in \mathbb{Z} \backslash\{0\} \mid\left(p_{k}, q_{k}\right) \neq(0,0)\right\}$. Then $u^{-k_{0}}\left(P(u)-p_{0}\right)$ and $u^{-k_{0}}\left(Q(u)-q_{0}\right)$ are linearly independent polynomials of the variable $u$. This is equivalent to the linear independence of the coefficients of $P-p_{0}$ and $Q-q_{0}$, that is, of $\left(p_{k}\right)_{k \in \mathbb{Z} \backslash\{0\}}$ and $\left(q_{k}\right)_{k \in \mathbb{Z} \backslash\{0\}}$. Therefore, the system of vectors $\left(p_{k}, q_{k}\right)_{k \in \mathbb{Z} \backslash\{0\}}$ spans $\mathbb{R}^{2}$. Thus there exists $\ell \in \mathbb{Z} \backslash\{0\}$ such that, for $0 \neq k<\ell$, the vector $\left(p_{k}, q_{k}\right)$ is parallel to $\left(p_{k_{0}}, q_{k_{0}}\right)$ and $\left(p_{\ell}, q_{\ell}\right)$ is not parallel to $\left(p_{k_{0}}, q_{k_{0}}\right)$. Then,

$$
p_{i} q_{j}=p_{j} q_{i} \quad(0 \neq i<\ell, 0 \neq j<\ell) \quad \text { and } \quad p_{k_{0}} q_{\ell} \neq p_{\ell} q_{k_{0}} .
$$

Now let $v \in I$ be fixed and $r \in(I / v) \cap \mathbb{Q}$ ). Then $u=r v \in I$, therefore, the equality (24), the additivity and $\mathbb{Q}$-homogeneity of $d$ imply

$$
\left(\sum_{j \in \mathbb{Z}} q_{j} j r^{j-1} v^{j-1}\right)\left(\sum_{i \in \mathbb{Z}} p_{i} r^{i} d\left(v^{i}\right)\right)=\left(\sum_{i \in \mathbb{Z}} p_{i} i r^{i-1} v^{i-1}\right)\left(\sum_{j \in \mathbb{Z}} q_{j} r^{j} d\left(v^{j}\right)\right) .
$$


Using that $d(1)=0$, this equality is equivalent to

$$
\sum_{i \in \mathbb{Z} \backslash\{0\}} \sum_{j \in \mathbb{Z} \backslash\{0\}} p_{i} q_{j} r^{i+j-1}\left(j v^{j-1} d\left(v^{i}\right)-i v^{i-1} d\left(v^{j}\right)\right)=0 .
$$

This implies

$$
\sum_{i<j, i j \neq 0}\left(p_{i} q_{j}-p_{j} q_{i}\right) r^{i+j-1}\left(j v^{j-1} d\left(v^{i}\right)-i v^{i-1} d\left(v^{j}\right)\right)=0 .
$$

According to the choice of $k_{0}$ and $\ell$, we have (26), therefore,

$$
\sum_{k_{0} \leq i, \max (i+1, \ell) \leq j, i j \neq 0}\left(p_{i} q_{j}-p_{j} q_{i}\right) r^{i-k_{0}+j-\ell}\left(j v^{j-1} d\left(v^{i}\right)-i v^{i-1} d\left(v^{j}\right)\right)=0 .
$$

The left hand side of this equality is a polynomial of $r$, hence its value at $r=0$ is equal to zero, which gives

$$
\left(p_{k_{0}} q_{\ell}-p_{\ell} q_{k_{0}}\right)\left(\ell v^{\ell-1} d\left(v^{k_{0}}\right)-k_{0} v^{k_{0}-1} d\left(v^{\ell}\right)\right)=0 .
$$

By the last relation in (26), this yields

$$
\ell d\left(v^{k_{0}}\right)=k_{0} v^{k_{0}-\ell} d\left(v^{\ell}\right) \quad(v \in I) .
$$

With the substitution $u:=v^{\ell}$, and with the notation $r:=\frac{k_{0}}{\ell}$, we get

$$
d\left(u^{r}\right)=r u^{r-1} d(u) \quad\left(u \in J:=\left\{x^{\ell} \mid x \in I\right\}\right) .
$$

Observe that $r \in \mathbb{Q} \backslash\{0,1\}$, therefore, by Lemma 4, it follows that $d$ is a standard derivation.

If condition (i) holds and $P$ is not identically zero, then $Q$ is a rational multiple of $P$, hence (24) is trivially valid by the $\mathbb{Q}$-homogeneity of $d$.

If condition (ii) holds, then, denoting $P_{0}:=P-p_{0}$ and $Q_{0}:=Q-q_{0}$ and using (i) for $P_{0}$ and $Q_{0}$, we have

$$
\begin{aligned}
& Q^{\prime}(u) d(P(u))=Q_{0}^{\prime}(u) d\left(P_{0}(u)+p_{0}\right)=Q_{0}^{\prime}(u)\left(d\left(P_{0}(u)\right)+p_{0} d(1)\right)=Q_{0}^{\prime}(u) d\left(P_{0}(u)\right) \\
& \quad=P_{0}^{\prime}(u) d\left(Q_{0}(u)\right)=P_{0}^{\prime}(u)\left(d\left(Q_{0}(u)\right)+q_{0} d(1)\right)=P_{0}^{\prime}(u) d\left(Q_{0}(u)+q_{0}\right)=P^{\prime}(u) d(Q(u)) .
\end{aligned}
$$

Finally, if condition (iii) is valid, i.e., $d$ is a standard derivation, then, for all $u \in I$ and $i, j \in \mathbb{Z}$,

$$
j u^{j-1} d\left(u^{i}\right)=j u^{j-1} i u^{i-1} d(u)=i u^{i-1} d\left(u^{j}\right) .
$$

Multiplying this equality by $p_{i} q_{j}$, then summing up the equalities so obtained side by side for $(i, j) \in \mathbb{Z}^{2}$, we obtain that

$$
\left(\sum_{j \in \mathbb{Z}} q_{j} j u^{j-1}\right)\left(\sum_{i \in \mathbb{Z}} p_{i} d\left(u^{i}\right)\right)=\left(\sum_{i \in \mathbb{Z}} p_{i} i u^{i-1}\right)\left(\sum_{j \in \mathbb{Z}} q_{j} d\left(u^{j}\right)\right) .
$$

This equality, by the additivity and $\mathbb{Q}$-homogeneity of $d$ is equivalent to (24).

Corollary 9. Let $d: \mathbb{R} \rightarrow \mathbb{R}$ be an additive function, I be a nonempty open interval not containing zero and assume that $P, Q: I \rightarrow \mathbb{R}$ are of the form (23), where $p_{k}, q_{k} \in \mathbb{Q}$ for all $k \in \mathbb{Z}$ and the set $\left\{k \in \mathbb{Z} \mid\left(p_{k}, q_{k}\right) \neq(0,0)\right\}$ is finite. Assume that $Q^{\prime}$ is non-vanishing on $I$, furthermore $P-p_{0}$ and $Q-q_{0}$ are linearly independent and d derivates $P \circ Q^{-1}$ on $J:=Q(I)$. Then $d$ is a standard derivation. 
Proof. Using that $d$ derivates $P \circ Q^{-1}$, we have

$$
d\left(P\left(Q^{-1}(v)\right)\right)=P^{\prime}\left(Q^{-1}(v)\right) \frac{1}{Q^{\prime}\left(Q^{-1}(v)\right)} d(v) \quad(v \in J) .
$$

Substituting $u:=Q^{-1}(v) \in I$ into the above equation, we can see that (24) holds. Taking into consideration that $P-p_{0}$ and $Q-q_{0}$ are linearly independent by assumption, Theorem 8 implies that $d$ is a standard derivation.

Corollary 10. Let $d: \mathbb{R} \rightarrow \mathbb{R}$ be an additive function, let $P$ be a nonzero real polynomial with rational coefficients and $I$ be a nonempty open subinterval of $\mathbb{R}$. Then $d$ derivates $P$ over $I$ if and only if

(i) either $\operatorname{deg}(P)=0$ and $d(1)=0$,

(ii) or $\operatorname{deg}(P)=1$ and $P(0) d(1)=0$,

(iii) or $\operatorname{deg}(P) \geq 2$ and $d$ is a standard derivation.

Proof. This statement is an immediate consequence of Theorem 8 by choosing $Q(u)=u$.

\section{Open Questions}

Motivated by the results of the previous section, we can formulate two open problems. Let $P, Q: I \rightarrow \mathbb{R}$ by of the form (23), where $p_{k}, q_{k} \in \mathbb{Q}$ for all $k \in \mathbb{Z}$ and the set $\left\{k \in \mathbb{Z} \mid\left(p_{k}, q_{k}\right) \neq\right.$ $(0,0)\}$ is finite and let $d: \mathbb{R} \rightarrow \mathbb{R}$ be an additive function.

Problem 1. Assume that $Q$ is non-vanishing on $I$ and $d$ derivates $P / Q$ on $I$, that is,

$$
d\left(\frac{P(u)}{Q(u)}\right)=\frac{P^{\prime}(u) Q(u)-Q^{\prime}(u) P(u)}{Q^{2}(u)} d(u) \quad(u \in I) .
$$

Under what conditions on $P$ and $Q$ does this equality imply that $d$ is a standard derivation?

Problem 2. Assume that $P^{\prime}$ is non-vanishing on $I$ and $Q(I) \subseteq P(I)$ and $d$ derivates $P^{-1} \circ Q$ on $I$, that is,

$$
d\left(P^{-1}(Q(u))=\frac{Q^{\prime}(u)}{P^{\prime}\left(P^{-1}(Q(u))\right)} d(u) \quad(u \in I) .\right.
$$

This, provided that $Q$ is strictly monotone on $I$, is equivalent to the condition

$$
P^{\prime}\left(P^{-1}(v)\right) d\left(P^{-1}(v)\right)=Q^{\prime}\left(Q^{-1}(v)\right) d\left(Q^{-1}(v)\right) \quad(v \in Q(I)) .
$$

Under what conditions on $P$ and $Q$ does this equality imply that $d$ is a standard derivation?

The result of Boros and Erdei [2] would be a particular case of such a generalization.

\section{REFERENCES}

[1] R. Badora, On approximate derivations, Math. Inequal. Appl., 9 (2006), 167-173.

[2] Z. Boros and P. Erdei, A conditional equation for additive functions, Aequationes Math., 70 (2005), $309-313$.

[3] Z. Boros and E. Gselmann, Hyers-Ulam stability of derivations and linear functions, Aequationes Math., 80 (2010), 13-25.

[4] Z. Daróczy and Gy. Maksa, Nonnegative information functions, Analytic function methods in probability theory (Proc. Colloq. Methods of Complex Anal. in the Theory of Probab. and Statist., Lajos Kossuth Univ. Debrecen, Debrecen, 1977), (North-Holland, Amsterdam, 1979), p. 67-78.

[5] W. Fechner and E. Gselmann, General and alien solutions of a functional equation and of a functional inequality, Publ. Math. Debrecen, 80 (2012), 143-154. 
[6] R. Grünwald and Zs. Páles, On derivations with respect to finite sets of smooth functions, Acta Math. Hungar. 154 (2018), no. 2, 530-544.

[7] E. Gselmann, Notes on the characterization of derivations, Acta Sci. Math. (Szeged), 78 (2012), 137-145.

[8] E. Gselmann, Derivations and linear functions along rational functions, Monatsh. Math., 169 (2013), 355370.

[9] E. Gselmann, Approximate derivations of order n, Acta Math. Hungar., 144 (2014), 217-226.

[10] E. Gselmann and Zs. Páles, Additive solvability and linear independence of the solutions of a system of functional equations Acta Sci. Math. (Szeged), 82 (2016), 101-110.

[11] F. Halter-Koch, A characterization of derivations by functional equations, Math. Pannon., 11 (2000), 187190.

[12] F. Halter-Koch, Characterization of field homomorphisms and derivations by functional equations, Aequationes Math., 59 (2000), 298-305.

[13] W. B. Jurkat, On Cauchy's functional equation, Proc. Amer. Math. Soc., 16 (1965), 683-686.

[14] M. Kuczma, An Introduction to the Theory of Functional Equations and Inequalities, Prace Naukowe Uniwersytetu Śląskiego w Katowicach, vol. 489, Państwowe Wydawnictwo Naukowe - Uniwersytet Ślạski, (Warszawa-Kraków-Katowice, 1985), 2nd edn. (ed. by A. Gilányi), (Birkhäuser, Basel, 2009).

[15] S. Kurepa, The Cauchy functional equation and scalar product in vector spaces, Glasnik Mat.-Fiz. Astronom. Društvo Mat. Fiz. Hrvatske Ser. II, 19 (1964), 23-36.

[16] S. Kurepa, Remarks on the Cauchy functional equation Publ. Inst. Math. (Beograd) (N.S.), 5 (19) (1965), $85-88$.

[17] Gy. Maksa, Deviációk és differenciák (Deviations and Differences), (in Hungarian), Phd thesis, (Lajos Kossuth University, Debrecen, Hungary, 1976).

[18] Gy. Maksa, On near derivations, Proc. Amer. Math. Soc., 81 (1981), 406-408.

[19] Gy. Maksa, On the trace of symmetric bi-derivations, C. R. Math. Rep. Acad. Sci. Canada, 9 (1987), 303-307.

[20] Gy. Maksa, On additive functions which differentiate elementary functions in some sense, Ann. Univ. Sci. Budapest. Sect. Comput., 41 (2013), 125-136.

[21] Gy. Maksa and Zs. Páles, Convexity with respect to families of means, Aequationes Math., 89 (2015), 161167.

[22] A. Nishiyama and S. Horinouchi, On a system of functional equations, Aequationes Math., 1 (1968), 1-5.

Institute of Mathematics, University of Debrecen, H-4002 Debrecen, Pf. 400, Hungary

E-mail address: richard.grunwald96@gmail.com, pales@science.unideb.hu 